\title{
Functional Preparedness of Women-Martial Artists
}

\author{
Akopyan A.O. \\ Federal Scientific Center Institute \\ Moscow, Russia \\ acop1@yandex.ru
}

\author{
Kulagina L.A. \\ Federal Scientific Center Institute \\ Moscow, Russia \\ Kulagina1@rambler.ru
}

\begin{abstract}
Objective: to conduct a comparative analysis of the of training functional reserve (FRT) at the stages of women-martial artists direct training for competitions. The research involved 27 athletes, aged 19 to 25 years, included in the main composition of candidates for participation in the European Championships. Testing of FRT of women-single combats (judo, Boxing, female wrestling) took place at stages of direct preparation for competitions. The comparative characteristic of preparation means, their volume and intensity is presented. The dynamics of the women-martial artists FRT depending on the volume and structure of training facilities at the stages of direct training for competitions is presented. It is shown that the rational distribution of training facilities corresponds to the maximum increase in FRT. The minimum increase in FRT was $7 \%$ (judo), the maximum - $12.2 \%$ (women's wrestling).
\end{abstract}

Keywords-boxing; judo; women's wrestling; preparedness; functional reserve of training; technique; team.

\section{INTRODUCTION}

Construction of training process in training for competitions in types of sports single combats, now, has some scientific-methodical and experimental validity. As a rule, there are 3 stages of training-the basic stage, the special stage and the stage of direct training. The construction of the basic training stage largely depends on the general requirements-the improvement of general and special physical training, the structure and organization of means and methods depending on the sport [1,2]. The stages of special training are aimed at the implementation of the accumulated functional and physical potential into the effectiveness of technical and tactical skills [3,4,5].

\section{RESULTS}

Separately we will stop at the stage of direct training for competitions since it is the most responsible stage connected with realization of the developed functionalphysical and technical-tactical potential in the conditions of the maximum knocking down factors action. Duration of direct training stage in types of sports single combats is 1418 days. Optimality of such duration of a stage was confirmed by practice of successful performances by national national teams on judo, boxing and wrestling.

The analysis of stages of direct training in female types of sports single combats showed that the maximum volume of direct preparation is performed by women in wrestling. As can be seen from the diagrams presented in figure 1 , in boxing and judo, almost the same is duration of the stage, $1731 \mathrm{~min}$ and $1716 \mathrm{~min}$, respectively. Duration of direct training stage for competitions in female wrestling is much more and makes $2669 \pm$ minutes. Т (мин)

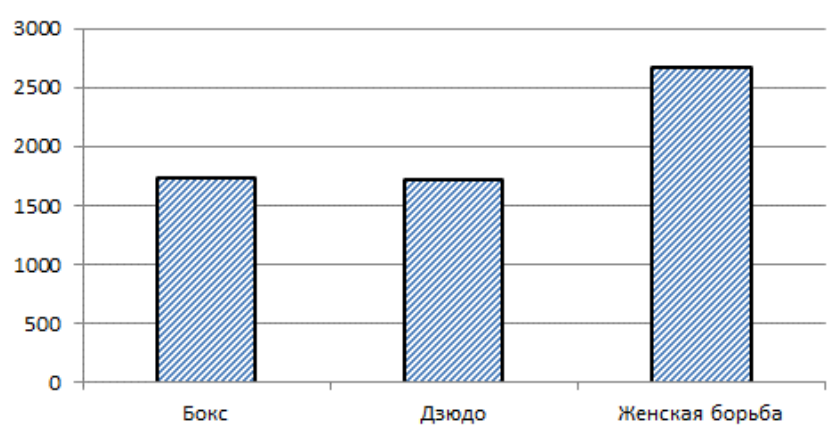

Fig. 1. Volume training work on the stages of women high class martial artists immediate training (min)

As it is visible from the presented volumes of training (fig. 1), the maximum and rather considerable volume of training work, in comparison with boxing and judo, is noted in female wrestling, differences make $45,0 \%$ - boxing and $45,6 \%$ - judo.

Since the main goal of the direct training stage is to bring the athlete to the maximum level of all sides of preparedness readiness, it is necessary to monitor and assess the functional state in the dynamics of the training process.

In practice of sports science there is sufficient number of instrumental control training process methods and athletes' organism adaptation to physical loads coaching received.

In our studies, to assess the functional state of the technique used to assess the functional reserve of training (FRT), method of FRT assessment developed in the center of martial arts VNIIFK (Hakobyan A. O., 2010, Russia).

The basis of the FRT calculation is based on the characteristics of the cardiovascular system, heart rate and blood pressure, carried out at rest and after a standard load (30 squats for 40). The calculation of FRT is performed automatically by a special computer program that allows to obtain digital and graphical information of 3 parameters: 
beginning and at the end of the direct training stage for the European Championships.

\section{CONCLUSION}

The analysis of FRT testing results at the beginning and at the end of direct training stage revealed that the maximum increase of FRT is $12,2 \%$ (female wrestling), minimum $7,0 \%$ is noted in judo. This fact allows to speak about greater efficiency of the training process construction in female wrestling.

The construction of the direct training stage in Boxing and judo requires scientific justification and appropriate correction, depending on the individual functional capabilities of candidates for the national team.

\section{References}

[1] Akopyan A.O. Express-assessment of the training functional reserve level in the types of martial arts. Sport Science Bulletin. 2008, 4, pp. 46.

[2] Filimonov V. I. Boxing. Sports-technical and physical training (monograph). M.: "Insan". 2000, 432.

[3] Jeroyan G.O., Khudadov N.A. Pre-Competition training of boxers. M.: FIS. 1971, 230.

Fig. 2. Distribution of training funds for the stages of immediate training for the Championships of Europe (\%)

Analyzing the data presented in figure 2, it should be noted the prevalence of FRT in all types of women's martial arts. It is connected with application of FRT means, as the means stimulating regenerative processes, after intensive training loadings of special and competitive training. It should be noted prevalence of volumes of training means of special $(44,5 \%)$ and competitive training $(17,2 \%)$ in judo.

As noted above, the assessment of the athletes' functional state was carried out by means of FRT, testing of which was carried out before the first morning training. Such statement of research allowed to estimate and, if necessary, to carry out correction of the subsequent training occupation. The correction was carried out at individual values of FRT $\leq 25.7 \%$. ed.

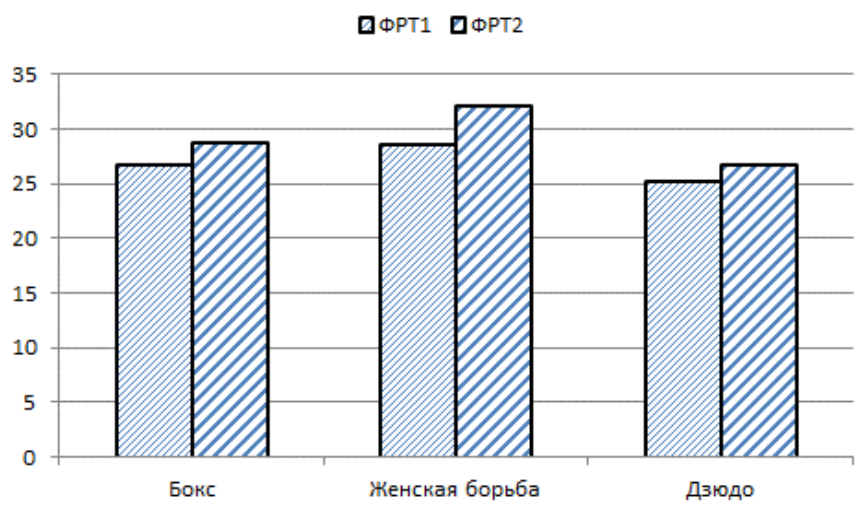

Fig. 3. Dynamics of FRT female martial artists at the beginning and end of the direct training phase

Comparative characteristics of the athletes' functional state are given by the average value of FRT indicators on the team. Fig. 3 shows the results of FRT testing at the
[4] Kuznetsov A., Mutaeva I., Kuznetsova Z. Diagnostics of functional state and reserve capacity of young athletes' organism. Proceedings of the $5^{\text {th }}$ International Congress on Sport Sciences Research and Technology Support. 2017.

[5] Alexsandr S. Kuznetsov, Sergei V. Novakovskiy, Oleg B. Solomakhin. About expediency of attack and defense parallel study in Greco-roman wrestling at the stage of initial training. The Russian journal of Physical Education and Sport. 2018, 13(1), pp. 12-20. DOI: 10/14526/01_2018_279. 